\title{
KIM-1 expression links kidney injury with CKD in mice
}

The connection between acute kidney injury and the development of chronic kidney disease (CKD) and end-stage renal disease is becoming increasingly apparent. Although the clinical importance of this interaction is known, the molecular details are not yet clear. Now, researchers led by Benjamin Humphreys have used mouse models to elucidate one of the molecular mechanisms underlying this link.

The researchers focused their attention on kidney injury molecule-1 (KIM-1) as it was established to be expressed transiently after acute kidney injury, as well as being chronically expressed in the fibrotic kidney. "We wanted to ask whether KIM-1 was actually playing a role in fibrosis, whether it was simply a biomarker of injury, or if alternatively it was driving a fibrotic reaction when expressed chronically," explains Humphreys.

Using a rodent model of renal fibrosisunilateral ureteral obstruction-the researchers observed that KIM-1 expression was strongly upregulated 2 days after the obstruction and then fell away. However, expression of KIM-1 remained significantly elevated 14 days after injury.

Although this information is interesting, a number of possible confounding factors exist that could affect the interpretation of the role of KIM-1 in kidney fibrosis. "To account for these factors, we used a genetic approach and created a mouse in which we could conditionally activate expression of KIM-1 in kidney tubules in the absence of any other injury stimulus," says Humphreys.

In their conditional transgenic model, expression of KIM-1 caused spontaneous and progressive interstitial kidney inflammation and fibrosis, even in the absence of any kidney injury. This finding was indicative of a role for KIM-1 in fibrosis. The researchers also used a mouse model with a truncated form of KIM-1, and showed that these mice were protected from fibrosis. Furthermore, expression of KIM-1 was associated with upregulation of the proinflammatory cytokine monocyte chemotactic protein-1 (MCP-1).

Taken together these data show that sustained expression of KIM-1 promotes kidney fibrosis, and provides a link between kidney injury and CKD. Humphreys points out that there is a lot more to learn: "It will be important to precisely define how KIM-1 is driving kidney fibrosis. We believe that upregulation of MCP-1 might be an intermediary. It will be equally important to determine whether KIM- 1 could be targeted therapeutically, with either small molecules or biologics, in order to assess whether such a strategy could help slow the progression of CKD in humans."

Rebecca Kirk

Original article Humphreys, B. D. et al. Chronic epithelial kidney injury molecule-1 expression causes murine kidney fibrosis. J. Clin. Invest. doi:10.1172/JCI45361 